\title{
Publisher Correction: Human heart-forming organoids recapitulate early heart and foregut development
}

Lika Drakhlis D, Santoshi Biswanath, Clara-Milena Farr, Victoria Lupanow, Jana Teske, Katharina Ritzenhoff, Annika Franke, Felix Manstein (D), Emiliano Bolesani, Henning Kempf D, Simone Liebscher, Katja Schenke-Layland (D), Jan Hegermann, Lena Nolte, Heiko Meyer, Jeanne de la Roche (D), Stefan Thiemann, Christian Wahl-Schott (D), Ulrich Martin (iD and Robert Zweigerdt (iD

Correction to: Nature Biotechnology https://doi.org/10.1038/s41587-021-00815-9, published online 8 February 2021.

This paper was originally published under standard Springer Nature license (@ The Author(s), under exclusive licence to Springer Nature America, Inc.). It is now available as an open-access paper under a Creative Commons Attribution 4.0 International license, (c) The Author(s), in accordance with the Nature Transformative Agreement for Germany. The error has been corrected in the print, HTML and PDF versions of the article.

licenses/by/4.0\%.
}

Published online: 20 May 2021

https://doi.org/10.1038/s41587-021-00960-1

(C) The Author(s) 2021 